\title{
Incorporating Advanced Microscopy Techniques into the Undergraduate Curriculum
}

\author{
C.B. Marks*
}

*University of Richmond, Department of Biology, 28 Westhampton way, Richmond VA 23173

One topic I think we can all agree on as microscopist, is the need to train and educate today's students in the area of advanced microscopy so that we have microscopy experts in the future. However, Microscopy is not for everyone. So, how do you reach the subset of students who may have an interest in microscopy? How do you recruit them into training? You take them as undergraduates, and give them hands-on exposure, the earlier in their studies, the better.

The students I instruct with hands-on microscopy range from highly supervised non-science majors to summer research students who can function independently. The goal of the experience varies with the skill set of the particular group invited into the laboratory. The purpose of incorporating microscopy into the curriculum is not to show off an expensive piece of equipment, but rather to illustrate how these machines can be used as tools to explore the subject matter being taught in the classroom. How this is accomplished depends on the subject matter, the course instructor, and the level of sophistication of the students. It really is not much different than working in a core facility, and having a new project come in.

In general, a few non-major classes and most first year biology courses take advantage of the Scanning Electron Microscope (SEM). The Transmission Electron Microscope (TEM) has been incorporated into a few courses at different levels, most notably the TEM has become a routine component of our Cell Biology course. We also have some upper level classes for our undergraduates that will introduce them to using our Laser Scanning Confocal Microscope (LSCM) as one tool of many for studying cells. Examples of projects include the differences in gram negative and positive cells (TEM); student preps of cultured bacterial samples (SEM); the effects of drugs on cell growth and cytoskeletal architecture (SEM and LSCM); Interpreting cell ultrastructural 'clues' to solve the mystery of cell type/function (TEM).

During my presentation, I will discuss the initial logistical problems, how they were addressed, and how advanced microscopy has expanded into the curriculum while I have been at University of Richmond. I will also go into more detail on what stays the same and what changes as students become more sophisticated in their abilities.

Hands-on student use of SEM, TEM, and LSCM can be used as teaching tools within the confines of undergraduate biology laboratories. In order to make this work, teamwork on the part of the instructors, and the students is essential. Concrete goals and expectations in the design and execution of the lab promote student ownership of the material, and more direct evaluation of student work by the instructors. With time, students who enjoy and excel in these curricular experiences become involved with summer research projects that use microscopy methods. Some of these students will go on to present their work at professional meetings, and some will also become co-authors of peer-reviewed articles based on research completed during their undergraduate years at University of Richmond. 